\title{
ProSUM: Prospecting Secondary raw materials in the Urban mine and Mining wastes
}

\section{Systematic harmonisation and classification of data sources for mapping EU secondary raw materials in Electronics, Batteries, Vehicles and Mining Wastes}

Jaco Huisman $^{* 1}$, Hina Habib ${ }^{1}$, Michelle Guzman Brechu ${ }^{1}$, Sarah Downes ${ }^{2}$, Lucia Herreras ${ }^{3}$, Amund N. Løvik ${ }^{4}$, Patrick Wäger ${ }^{4}$, Daniel Cassard ${ }^{5}$, François Tertre ${ }^{5}$, Paul Mählitz ${ }^{6}$, Susanne Rotter ${ }^{6}$, Perrine Chancerel ${ }^{6}$, Maria Ljunggren Söderman ${ }^{7}$

${ }^{1}$ United Nations University, Bonn, Germany

${ }^{2}$ Repic Ltd, Greater Manchester, United Kingdom

${ }^{3}$ WEEE Forum, Brussels, Belgium

${ }^{4}$ EMPA, St. Gallen, Switzerland

${ }^{5}$ BRGM, Orléans, France

${ }^{6}$ Technische Universität Berlin, Germany

${ }^{7}$ Chalmers University of Technology, Sweden

* Corresponding Author, Huisman@unu.edu, +31 626664780

\begin{abstract}
ProSUM - Latin for "I am useful" - aims to provide better information on raw materials from secondary origins. It focuses in particular on the content of Critical Raw Materials (CRMs) from Batteries (BATT), Waste Electrical and Electronic Equipment (WEEE), End of Life Vehicles (ELV) and Mining Wastes (MIN) available for processing in Europe. However, data for these products are usually very scattered amongst a variety of institutions, including government agencies, universities, NGOs and industry. This deficit is addressed in this H2020 funded project. ProSUM will establish a European network of expertise on secondary sources of CRMs, vital to today's high-tech society. It coordinates efforts to collect secondary CRM data and collate maps of stocks and flows for materials and products in the "urban mine". The project will construct a comprehensive inventory identifying and mapping CRM stocks and flows across the European Union (EU). Via a user-friendly, open-access Urban Mine Knowledge Data Platform (EU-UMKDP), it will combine and relate them to primary raw materials data from the EU-FP7 Minerals4EU project and communicate the results online through the future European Geological Data Infrastructure (EGDI) at large. It will also provide update protocols, standards and recommendations to maintain and expand the EUUMKDP in the future.
\end{abstract}

\section{Introduction}

This article highlights the outcomes of the critical first steps in the project: to produce a system for defining, harmonising and classifying data for all relevant products, waste streams and their composition. CRM data are often stored in a variety of databases (DBs) with their own design and vocabulary, making any attempt to merge them difficult. Problems related to availability, quality, organisation, accessibility and sharing of these structured data are common to a large number of organisations and public authorities in Europe. Data for EEE, BATT and ELV are also frequently presented in unstructured sources such as academic literature, technical reports and individual data points. Here the temporal and geographic scope, assumptions, data representativeness and data quality are not described in a harmonised way, if at all. These unstructured sources are similarly problematic and require measures to allow exchange, sharing, access and use of interoperable (spatial) data and services, in order to produce a more consistent knowledge base at national and European levels. This requires correlation with published statistics and recognised classification systems as well as a harmonised approach for collating and aggregating data from other 'unstructured' sources. Data in the knowledge base has to be harmonised in order to produce the highest quality data for the inventory. Moreover, harmonisation is required in order to combine data protocols with automated data harvesting systems. Therefore, both available and newly developed classifications are described in correlation tables and specific recommendations are made available for other researchers.

\section{The EU-UMKDP}

The ProSUM unified data model for WEEE, BATT and ELV and the INSPIRE MR data model, related to Mining Waste, organises data elements and standard- 


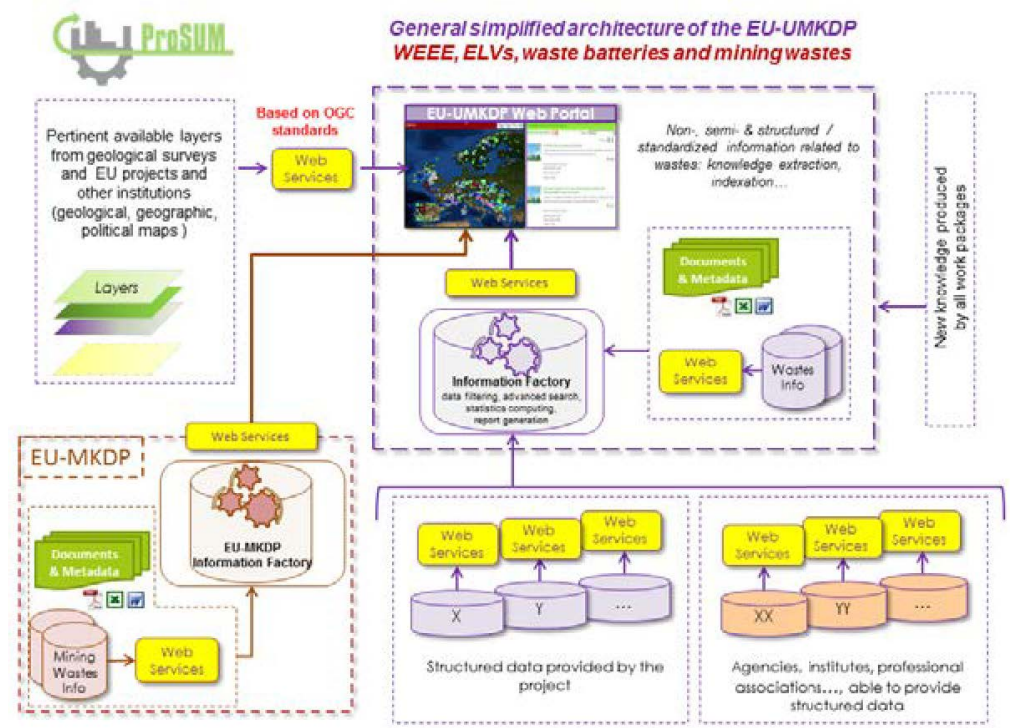

Figure 1 - Simplified architecture of the future EU-UMKDP

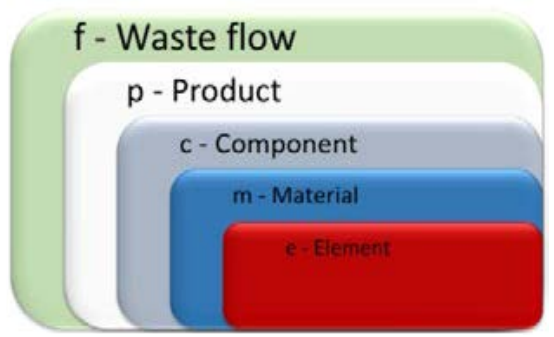

Figure 2 Simplified ProSUM calculation sequence ises how they relate to one another. The INSPIRE Directive lays down a general framework for a Spatial Data Infrastructure (SDI) for the purposes of European Community environmental policies or activities which may have an impact on the environment [8]. The INSPIRE MR data model is the European approved data model for mineral resources (MR), including both primary and secondary (i.e., Mining wastes) resources. However, mining wastes do not belong to the core part of this data model, being only an extension. One objective of the ProSUM project is to improve and extend the mining wastes part of the INSPIRE MR data model as well. The ProSUM unified data model will describe the structure, manipulation and integrity aspects of the urban mining data stored in the EU - UMKDP and in data management systems in general [14]. Figure 1 illustrates how the final delivery from the project, the EU - UMKDP (Urban Mine Knowledge Data Platform) system is designed to accommodate both structured data from existing databases (data produced by external providers and/or synthesised by project partners) and semi- or unstructured information produced by ProSUM work packages (reports, notes, publications and presentations).

Figure 2 provides a simplified illustration of the ProSUM calculation sequence in order to determine the composition of WEEE, BATT and ELV. In some studies, the product composition is given directly at the elemental level (chemical composition), but often the data is only available for selected components (e.g., the hard disk of a personal computer) or at a material level. As an example, aluminium metal usually refers to a metallic alloy containing up to $25 \%$ of other elements, rather than the pure element aluminium. The so-called CRM parameters are given as a single letter or a combination of two letters as illustrated in Figure 2. A single letter indicates data with an extensive property for the entity. For example, data on the mass of dishwashers might be of interest, and would be labelled ' $p$ ' for product. It is also possible to provide other extensive properties, such as

\begin{tabular}{|c|c|c|c|}
\hline \multicolumn{4}{|c|}{ Stocks and Flows (f) } \\
\hline $\begin{array}{c}\text { Product } \\
\text { Flow Type }\end{array}$ & $\begin{array}{l}\text { Stock } \\
\text { Type } W\end{array}$ & $\begin{array}{l}\text { Vaste Generation } \\
\text { Types }\end{array}$ & $\begin{array}{c}\text { Waste Flow } \\
\text { Types }\end{array}$ \\
\hline \multicolumn{4}{|l|}{ Products (p) } \\
\hline $\begin{array}{c}\text { UNU Key } \\
(54)\end{array}$ & BATT key & ELV key & $\begin{array}{c}\text { Vehicle Engine- } \\
\text { Power Type }\end{array}$ \\
\hline $\begin{array}{l}\begin{array}{l}\text { UNU Sub- } \\
\text { key }(>180)\end{array} \\
\end{array}$ & $\begin{array}{c}\text { BATT sub- } \\
\text { key (16) }\end{array}$ & Vehicle Type & $\begin{array}{c}\text { Vehicle } \\
\text { Weight Class } \\
\end{array}$ \\
\hline $\begin{array}{c}\text { Device Type } \\
(>700)\end{array}$ & & \begin{tabular}{|c|} 
Vehicle \\
Segment Type
\end{tabular} & \\
\hline
\end{tabular}

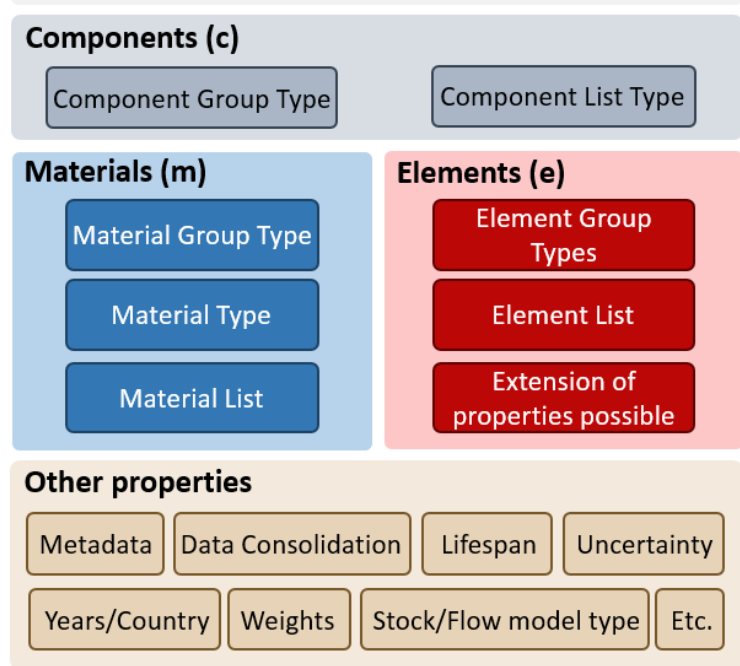

Figure 3 Simplified ProSUM data organisation 
the area of an integrated circuit, which would be labelled ' $c$ ' for components. A combination of two letters indicates an extensive or intensive property (usually mass or mass fraction) of an entity in another entity. An example is 'e-c', which indicates that the data is on the total mass of an element per component in a specific Unit of Measure (UoM) e.g. g/piece or as a mass fraction (e.g., $\mathrm{mg} / \mathrm{kg}$ ) of an element in a component. The current list of CRM parameters used the most is: 'e-m' for the mass or mass fraction of an element in a material; 'e-c' for the mass or mass fraction of an element in a component; 'e-p' for the mass or mass fraction of an element in a product; ' $\mathrm{m}$ c' for the mass, mass fraction or volume of a material in a component; ' $m$-p' for the mass, mass fraction or volume of a material in a product; 'c-c' for the mass, mass fraction, number, length, volume, area or other extensive property of a component in another component; 'c-p' for the mass, mass fraction, number, length, volume, area or other extensive property of a component in a product; 'p-p' for the mass, mass fraction, number, length, volume, area or other extensive property of a product in another product; and finally; 'p-f' for the mass, mass fraction or number of pieces of products in a stock or flow. Note that 'c-c', and 'p-p', are also valid options, since a product (e.g., battery) may sometimes appear as a component of other products, and a component can often be subdivided into other components. For example, a populated printed circuit board and its capacitors are both considered components in this system. Based on this CRM characterisation, Figure 3 provides a simplified view of the resulting ProSUM data organisation. A key question here is the feasibility of doing the calculations mainly as a multiplication of 'e-m', 'm-c' and 'c-p', especially because components and materials are often treated as the same "layer" in practice. Here the most desired route in ProSUM is going from ' $e-m$ ' to ' $m-c$ ' to 'c-p' with data for all these points, where possible. If there is a lack of data, the data consolidation aims at storing at least one selected data-point for instance for ' $e-p$ ' for each product key. In order to enable the above system to function, and more importantly, to be updated and maintained in the future, there are three key tasks in ProSUM that need to be structured, defined and harmonised as clearly and unambiguously as possible:

1. Clear definitions for every data model part;

2. Uniform classifications and correlation tables;

3. Harmonised descriptions of data quality, uncertainty and the handling of metadata.

\section{Definitions}

For all parts of the ProSUM unified data model, definitions are adopted from respective EU legal texts, other relevant sources and where not available, established within the project. In Figure 4 an overview of the most relevant definitions is provided. Within the ProSUM database, 'components', 'materials' and 'elements' are included but 'substances' are not, since so-called Full Material Declarations (FMD) are rarely 'full' complete data sets. 'Components' are defined as uniquely identifiable parts or sub-units of products. They are usually mechanically removable in one piece and are considered indivisible for a particular function or use. The term 'Materials', as used here, refers to 'engineered materials' that are composed, manufactured and processed to achieve specific properties. Material properties are determined by the chemical composition and the necessary processing conditions. The material classification allows for assessment of the main matrix where CRMs are incorporated and also allows, in some cases like alloys, to an assessment of the CRM content. This information is important in order to quantify potential recycling materials. The term 'Elements' describes the

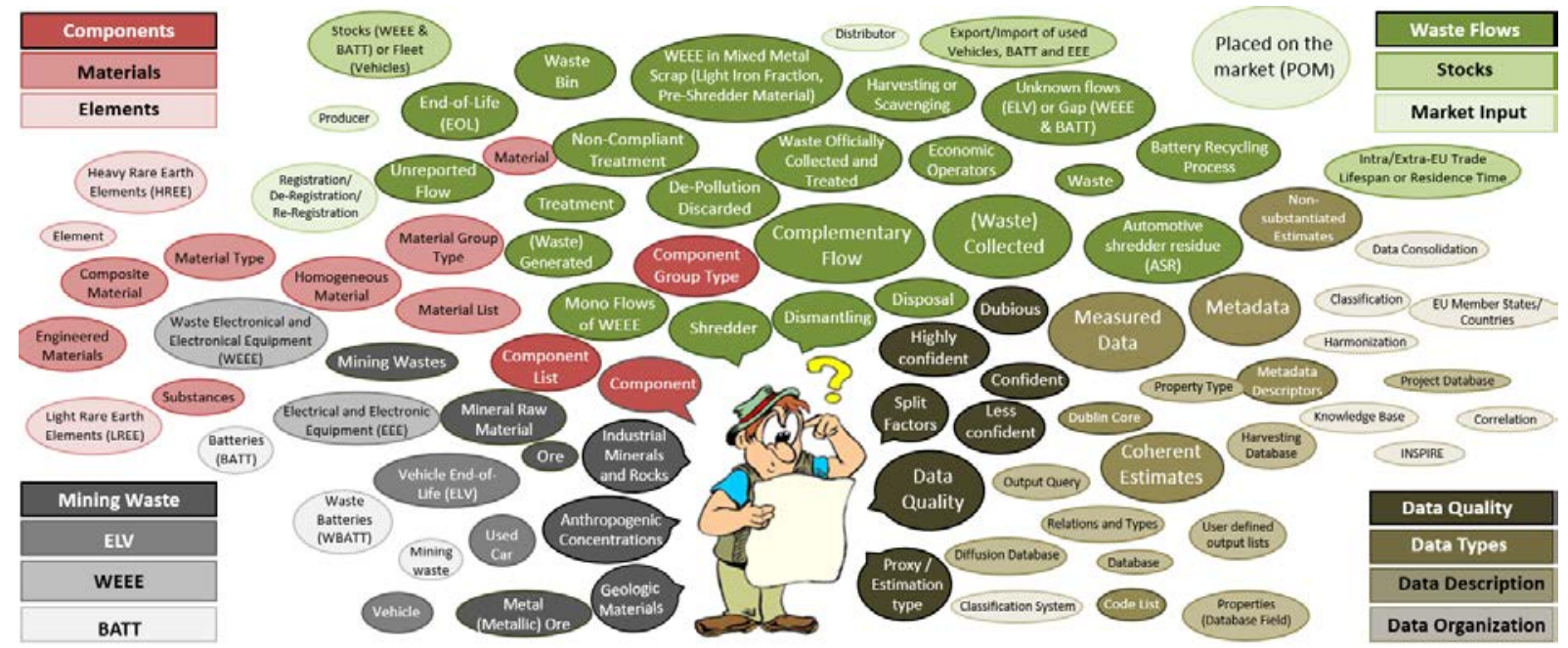

Figure 4, The ProSUM 'Definitions Cloud' 
elemental composition of products or components without considering their chemical speciation and follows the periodic table of elements. All definitions are available in [1].

\section{Classifications}

\subsection{Classification of stocks and flows}

Figure 4 shows the complexity of terms and definitions in use. For example, definitions regarding waste flows are few except those provided in the ELV, Battery and WEEE Directives on for instance 'treatment', 'recycling' and 'disposal'. Complementary flows are by definition not reported at a national level. These flows also include waste flows to noncompliant treatment e.g., recycled with other waste streams such as WEEE with mixed metal scrap which does not always meet the same efficiency and treatment standards, or Business to Business (B2B) waste collected and recycled but not reported to producer compliance schemes [7]. The amount of WEEE and BATT treated or not treated is very difficult to quantify. From a classification point of view, besides the 10 product and 6 collection categories of the original and recast WEEE Directives, other classifications are commonly used like Basel Convention Codes (Annex VIII), Custom codes and the EU List of Waste (LoW). These lists are not correlated, except for the LoW and Basel codes for which a correlation table exists. For ELV, the complementary flows are referred to as unknown whereabouts of vehicles. These are vehicles in use, hibernated or discarded, which are neither reported or registered as part of the European member states' (MS) vehicle fleets, nor as vehicles or ELVs exported from the EU. They include both legal and illegal activities and suboptimal end-of-life (EOL) vehicle registration. Where different classification systems exist, it is necessary to develop correlation tables to be able to compare, collate and aggregate data between different sources. See [1] and www.prosumproject.eu for more detailed information. Information on classifying various mass flow analysis models is also available, following the approach of [13].

\subsection{Classification of products}

\subsubsection{EEE: UNU Keys}

The classification of 'UNU keys' was developed by the United Nations University (UNU) and envelops all possible Electrical and Electronic Equipment (EEE). The UNU keys are constructed such that product and waste groups share comparable average weights, material composition, EOL characteristics and lifespan distributions. This classification list is divided into 54 categories by linking all possible $\mathrm{W}(\mathrm{EEE})$ items (about 700 products) to various conventional categorisations. In a similar manner, the link between statistical codes has been developed aligning the classifications applied in trade statistics (the Harmonised System) and WEEE directive [2]. UNU keys (4 digits code) are split into UNU sub-key level (6 digits code) in order to provide greater granularity for product average weights and their lifetime profiles to forecast trends, sales and waste generation accordingly. However, some of the Combined Nomenclature $(\mathrm{CN})$ codes are often ambiguous as they do not include devices types covered under these codes. In practice, certain devices can and are declared under multiple codes. Therefore, direct relations between sub-keys and $\mathrm{CN}$ codes can have scoping risks. Another list level covers over 700 device types ( 8 digits code). However, the device type list cannot be exhaustive as new appliance types enter the market continually. Correlation tables for all UNU keys, sub keys and device types are provided in [1]. The concept of UNU keys is also adopted by other international organisations as a basis for the statistical framework for measuring e-waste globally [6], as well as in the common methodology study for the European Commission for measuring amounts Placed on Market POM and WEEE generation [10].

\subsubsection{BATT Keys}

Batteries are used in products or connected to products as uninterrupted power supplies. They can be sold separately, be embedded in vehicles and EEE, or within the EEE embedded in vehicles. Several classification approaches for batteries exist, depending on cell chemistry, hazardousness, chargeability, and area of application. However, unlike the UNU keys, no well-structured classification existed until this project. Based on expert knowledge on battery systems and the materials they contain, as well as an analysis of existing battery classifications, a version of the ProSUM battery classification based on electrochemical cells was developed in the project [4]. The battery types cover the six current main electrochemical systems based on lithium, zinc, nickel-cadmium, nickel-metal hydride, lead and others. The six battery types are further divided into 16 BATT keys which will be used to consolidate data in a dedicated project database. The keys are classified by chargeability type, the Battery Directive descriptions, battery recycling flows and other trade codes such as the EU List of wastes, and $\mathrm{CN}$, and the United Nations Committee of Experts on the Transport of Dangerous Goods [1,4].

\subsubsection{Vehicle Keys}

In contrast to the classification of EEE, there is little previous work to build on for the classification of vehicles. The classification needed to be developed 
completely within the project. For efficiency, all vehicles addressed in the EU ELV Directive (vehicles below 3.5 tonnes) are within the scope. The starting point for the classification of vehicles is to define types of vehicles, materials and components in such a way that high masses and/or mass fractions of prioritised elements can be distinguished [5]. On the product level, vehicle type, vehicle powertrain and vehicle mass categories are defined based on existing regulations and reported in Eurostat data. It is known that vehicle equipment level is a determinant of the presence of prioritised elements, which may to some extent be captured by the vehicle segment categories commonly used in the automotive industry. However, no strict definition of vehicle segments exists, which is why cylinder size, also reported in Eurostat data, is chosen instead as a proxy for the vehicle segments. Subsequently, a classification system is proposed describing all possible combinations of vehicle type, vehicle powertrain and vehicle weight classes, as well as a cylinder size (as proxy for vehicle segment category). This approach allows a range of end user queries to be answered. See [1] and www.prosumproject.eu for all lists and a public consultation form for providing feedback on the proposed classification

\subsection{Classification of components}

Complex products consist of a number of highly specialised components that differ due to their function in material and elemental composition. CRMs are often concentrated in specific components, so called hotspots. In addition to that, many components in the recycling economy are already traded as secondary raw materials with high relevance due to the high content of CRMs such as printed circuit board, batteries, cables, etc. Component lists aim at describing consolidated product properties on a component level and identifying CRM hotspots. They are structured in a hierarchical way in order to allow the incorporation of higher aggregated data without losing more detailed information where available. All components are product specific, but also include components occurring in more than one product group, which is denoted by linkages to the respective product group.

These relational databases can be realised by creating tables which use the code list entries as input. Key sources of information on both new and waste products used in the ProSUM component lists are from legislative references, product data sheets, treatment recommendations, individual projects and disassembly studies plus from consultation with ProSUM partners to supplement information directly obtained from data holders like producer compliance organisations [1].

\subsection{Classification of materials and elements}

The material list from Figure 3 has three levels aggregating the entries from the list of materials 'Material List' in higher levels. From a top-down perspective, the 'Material Group Type' defines the main categories in which materials can be clustered as metals, polymers, ceramics, composites and biomass (based materials). Classification of materials uses: existing basic lists from materials science and from Life Cycle Assessment (LCA) databases, where the distinction between material, substance or chemical compounds is often not coherent and poorly structured; lists from the Minerals4EU Project which is "INSPIRE compliant"; and lists present in national and international standards like the European Norm (EN) or International Organisation of Standardization (ISO) which classify various materials by their composition and physical properties. The current materials list used in ProSUM is also expanded to include chemical elements relevant under the ProSUM scope.

\section{Harmonisation}

\subsection{Harmonisation of metadata de- scriptors}

Particularly for unstructured data, metadata information will be catalogued using fields based on Dublin Core descriptors [6]. Importantly, these metadata descriptors need harmonising within the entire consortium to describe the consolidated data in the databases and to share and collate bibliographical files. However, because of the expected multitude, variety and complexity of the data sources, additional fields are added to build, maintain and manage the ProSUM bibliography and Knowledge Base. Moreover, the descriptors used in the Dublin Core are in some cases open to interpretation. Therefore, a more precise description and definition is developed. Based on this, an interactive bibliography file is created containing about 420 individual sources on CRMs, mainly from scientific publications forming the Knowledge Base in the ProSUM portal (see section 6.2). The bibliography also provides a unique metadata ID, which is used throughout all project databases. See www.prosumproject.eu and [1] for all lists. For both ProSUM consortium members and others, a manual is created to ensure all data input providers classify their data sources in the same way. For structured data, the so-called 'MICKA' catalogue is used as a tool for structured data [12]. The MICKA catalogue has been proved for primary and secondary resources structured data in Minerals4EU and allows for the description of consolidated sources. 


\subsection{Harmonisation data quality (DQ)}

\subsubsection{Three types of information}

It is also important to establish a uniform method to evaluate the quality and reliability of data across all the three product groups, in particular where uncertainty levels have been used in lieu of information on standard deviations or confidence intervals. The approach is taken to clearly distinguish between three types of information: i) sources containing actual measured data; ii) sources based on coherent estimates and extrapolations; and iii) sources with expert assumptions and extrapolations which are insufficiently substantiated. Data of this third type is included in the Knowledge Base, but excluded from the analysis. In addition, comments related to the data quality assessment; the description of data consolidation steps where applicable; and the type of estimation used to produce a coherent estimate e.g., extrapolation, interpolation, is also stored in a harmonised way.

\subsubsection{Four data quality levels}

For describing data quality of all sources, the following four data quality levels are used [11]:

A = Highly confident: This level is defined as estimates or values based on a large number of measurements made from a large number of sources that represent a large part of the population.

B = Confident: A value based on a number of measurements made at a small number of representative samples, or an engineering calculation/ estimate based on a number of relevant facts and data points.

$\mathbf{C}=$ Less confident: A value based on a single measurement or an engineering judgement or estimate derived from a number of relevant facts and some assumptions.

D = Dubious: An estimate or value based on an engineering judgement derived from assumptions only or from a very limited number of data points.

Important to note is that for the previous first two types of information it can be the case that coherent estimates are of better quality than measured data. Secondly, in cases of multiple sources with different data quality for the same data point, the use of combination factors is likely to be used. For example for sources with data quality $\mathrm{C}-\mathrm{A}$, the final factor used is then $\mathrm{B}$ for the consolidated data point.

\subsubsection{Criteria for determining DQ}

Obviously, well-structured sources of data with associated representative statistical information are preferred. However, this is limited to a number of sources where clear specifications of the scope and data quality parameters are available. Hence, a common approach for judging data quality is needed based on unambiguous criteria:

- Is there a clear and consistent definition of the (product) scope and is temporal, geographic and demographic representativeness well specified?

- Are the sample size, assumptions and limitations to the data well described?

- Are there alternative sources (partly) confirming the data? Does the data fit into the time series, when available?

- When the data points are derived from a model: what is the scale (reach?) of the model and is the model validated?

- How many assumptions, estimates and proxies are made and how far reaching are they?

For each part of Figure 3, specific DQ criteria are in development for flows ('f'), products (' $p$ ') and for compositions ('c,m,e') both for literature sources and for actual chemical analysis measurements specifically (see the ProSUM data quality poster [9] for more information). In addition, within the project, internal review of these judgements is applied to minimise different interpretation levels [1].

\subsubsection{Weighting for multiple sources}

Where multiple sources are available for the same data point or coherent estimate, weighting is applied as indicated below to find the "best estimate" to ensure highly confident data sources which are prioritised.

\begin{tabular}{|l|l|}
\hline Data Quality Weight & Weight \\
\hline Highly confident & 4 \\
\hline Confident & 3 \\
\hline Less confident & 2 \\
\hline Dubious & 1 \\
\hline
\end{tabular}

Table 1 Data Quality Weight for multiple sources

\subsection{Harmonisation of Uncertainty (Unc) and error propagation}

\begin{tabular}{|l|l|l|}
\hline $\begin{array}{l}\text { Data Quality } \\
\text { Types }\end{array}$ & $\begin{array}{l}\text { Ranges for qualitative } \\
\text { judgement (flows) }\end{array}$ & Mean \\
\hline Highly confident & $0-10 \%$ & $5 \%$ \\
\hline Confident & $>10-20 \%$ & $15 \%$ \\
\hline Less confident & $>20-50 \%$ & $35 \%$ \\
\hline Dubious & $>50 \%$ & $100 \%$ \\
\hline $\begin{array}{l}\text { Data Quality } \\
\text { Types }\end{array}$ & $\begin{array}{l}\text { Ranges for qualitative } \\
\text { judgement (compositions) }\end{array}$ & Mean \\
\hline Highly confident & $0-20 \%$ & $10 \%$ \\
\hline Confident & $>20-50 \%$ & $30 \%$ \\
\hline Less confident & $>50-100 \%$ & $75 \%$ \\
\hline Dubious & $>100 \%$ & $200 \%$ \\
\hline
\end{tabular}

Table 2 Uncertainty levels for qualitative sources

In addition to data quality descriptions for all sources and data points, uncertainty levels are extracted from the original sources where available. The purpose of 


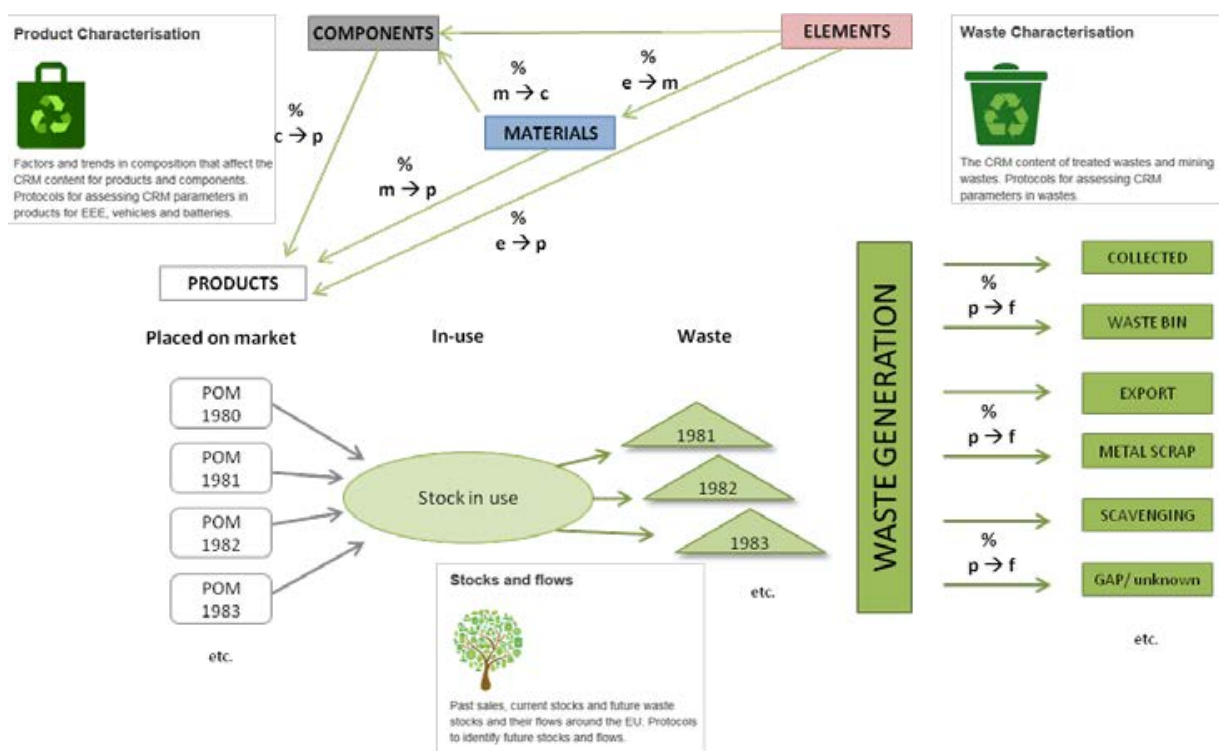

Figure 5 The ProSUM data model (example for WEEE)

this is to be able to determine the total uncertainty or error propagation of the values generated in the EUUMKDP from multiplications between the various data ('f,p,c,m,e') levels and for internal analysis. For quantitative sources with their statistical information, the ProSUM data model allows for various units of measurement for uncertainty. However, for sources without statistical information, the uncertainty ranges need to be estimated to allow an assessment of the total uncertainty ranges when both types of sources are combined. The qualitative judgement of Table 2 is used with distinct values for 'composition' versus 'stocks and flows'. For more information, see [9].

\section{Conclusions}

Using the above principles for the EU-UMKDP architecture [14] and the implementing rules of the

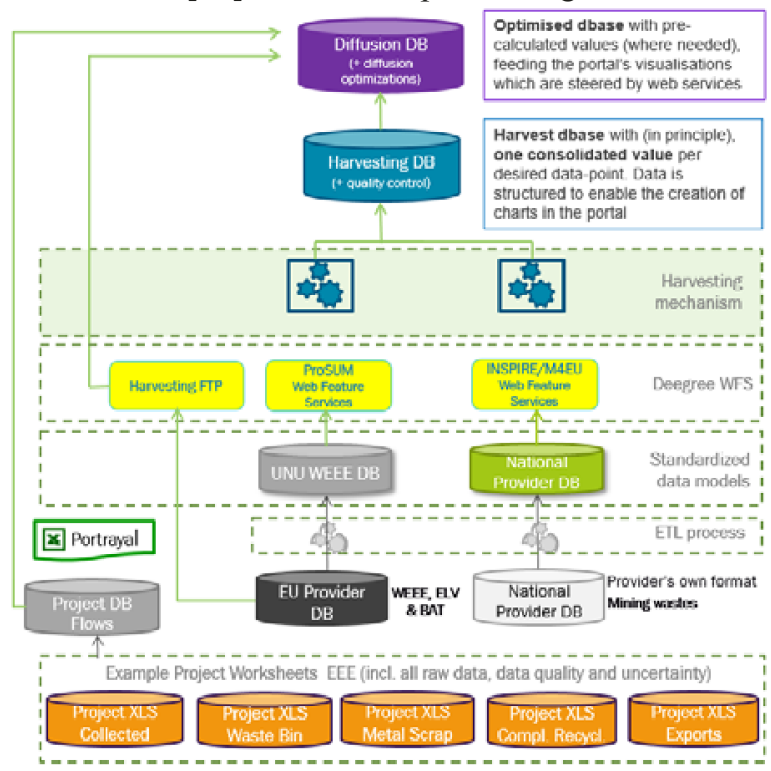

Figure 6 ProSUM Data Organisation
European INSPIRE Directive to make data and services interoperable across Europe, a single unified data model for WEEE, ELV and BATT products and their stocks, flows and composition is developed. Figure 5 shows the ProSUM schema for WEEE as an example. It builds on what already exists (the UNU database and associated data model [2,3]) and is expanded with all structured and unstructured information for as far it has been transformed (encoded) in structured information in the project. The figure also indirectly highlights the fundamental role of clear definitions for all available information (Section 3) as well as the data organisation itself. Secondly, the crucial role of having classifications established as almost every calculation step in Figure 5 builds on correlation tables allowing transformation of data (Section 4). Some of these tables will need to be updated annually. Finally, harmonisation of metadata descriptors, data quality and uncertainty levels is crucial to have a consistent approach where all calculations steps combined in Figure 5 deliver very interesting cross-sectoral final results on CRMs for various countries, products groups and years. Correlation tables also simplify so-called Extract Transform and Load process (ETL) which will be automated as much as possible in order to minimise human intervention, for future maintenance of the system. The structuring of data feeding the UMKDP is displayed in Figure 6. ProSUM-designed Web Feature Services or Excel Portrayals in the form of existing project databases will either enter directly into the Diffusion database through an FTP server or through harvesting databases based on the ProSUM unified data model. It should be noted that all structured information is often accompanied by unstructured information and related metadata in order to grant full access to all available information. 
In summary, based on the ProSUM unified data model and data organisation (Figures 5 and 6), the EUUMKDP under development will host the following services [14]:

- Targeted data visualisations and maps of the Urban Mine;

- A metadata catalogue for the delivery of relevant external spatial layers e.g., geological, geographical at both EU and national levels. This metadata catalogue will be accessible via an INSPIRE discovery service and a map viewer for the geographic representation of all spatial data;

- An information management system for all nonstructured information. This system will provide the user with the possibility to add semi- or unstructured documents to the Knowledge Base and data platform by creating metadata records. It will feed a search engine for all data, layers and documents delivered by the project and all external pertinent documents.

It is important to note the benefits of a harmonised EU wide data analysis on CRMs in the "urban mine". It provides an improved knowledge base, designed to aid and inform policy makers, industry, and research, to enhance resource efficiency of secondary raw materials in the EU. The detailed contents of the ProSUM harmonisation efforts [1] will be made available at http://www.prosumproject.eu/urban-mineknowledge-data-platform-work-underway including stakeholder consultation forms to provide feedback. In October 2016 an Information Network event is scheduled to discuss the ProSUM approach.

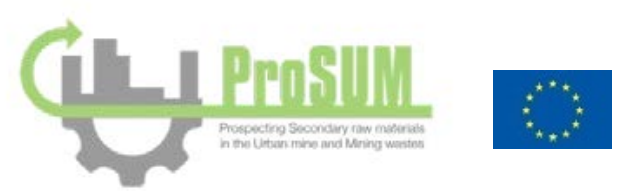

This project has received funding from the European Union's Horizon 2020 research and innovation programme under grant agreement No. 641999

\section{Literature}

[1] Straalen,V. van, Huisman, J., Habib, H., Chancerel, P., Maehlitz,P., Rotter, V.S., Wäger, P., Schjøth, F., Hallberg, A., Scheepens A., Cassard, D., Review and harmonisation of data, ProSUM Deliverable 5.3, available for public consultation in October 2016.

[2] Baldé, C. P., Kuehr, R., Blumenthal, K., Gill, S. F., Kern, M., Micheli, E., Magpantay, E., Huisman, J., E-waste Statistics: Guidelines on classifications, reporting and indicators, 2015.

[3] Baldé, C. P., Wang, F., Kuehr, R., Huisman, J., The Global E-Waste Monitor - 2014, 2015
[4] Chancerel, P., Maehlitz, P., Chanson, C., Binnemans, P., Huisman, J., Guzman Brechu, M., Nissen, N., Lang, K.D., Flows and Stocks of Critical Materials in Batteries: Data Collection and Data Uses, Proceedings Electronics Goes Green $2016+$.

[5] Du, X., Restrepro, E., Widmer, R. and Wäger, P., Quantifying the distribution of critical metals in conventional passenger vehicles using inputdriven and output-driven approaches: a comparative study. J. Mater. Cycles Waste Management, 218-228, 2015.

[6] Dublin Core Metadata Initiative. DCMI Specifications. Retrieved 09 17, 2015, from Metadata http://dublincore.org/specifications/

[7] Huisman, J., Botezatu, I., Herreras, L., Liddane, M., Hintsa, J., Luda di Cortemiglia, V., Leroy, P., Vermeersch, E., Mohanty, S., van den Brink, S., Ghenciu, B., Dimitrova, D., Nash, E., Shryane, T., Wieting, M., Kehoe, J., Baldé, C.P., Magalini, F., Zanasi, A., Ruini, F. and Bonzio, A. Countering WEEE Illegal Trade (CWIT), Summary Report, Market Analysis, Legal Analysis, Crime Analysis and Recommendations Roadmap. 2015

[8] INSPIRE Thematic working group Mineral Resources. D2.8.111.21 INSPIRE Data Specification on Mineral Resources - Draft Technical Guidelines. INSPIRE Thematic working group Mineral Resources, 2013.

[9] Løvik, A. N., Haarman, A., Scheepens, A., Rösslein, M., Wäger, P., Huisman, J., Prospecting Secondary Raw Materials in the Urban Mine: Data Quality and Uncertainty of Product Compositions, Proceedings Electronics Goes Green $2016+$.

[10] Magalini, F., Wang, F., Huisman, J., Kuehr, R., Baldé, C. P., Straalen, V. v., Hestin, M., Lecerf, L., Sayman, U. and Akpulat, O. Study on collection rates of waste electrical and electronic equipment (WEEE), 2015

[11] McInnes, G., Atmospheric Emission Inventory Guidebook, First Edition, A joint EMEP. CORINAIR Production, European Environment Agency, 1996.

[12]MICKA catalogue for ProSUM, seehttp://prosum.geology.cz/, Accessed 2016-07-25

[13] Wang, F., Huisman, J., Stevels, A. and Baldé, C. P. Enhancing E-waste estimates: improving data quality by multivariate Input-Output Analysis. Waste management, 33(11), 2397-2407, 2013.

[14] Cassard, D., Tertre, F., Schjøth, F., Heijboer, T., Čápová, D., Hallberg, A., Šinigoj, J., ProSUM EU-Urban Mining Knowledge Data Platform Specifications, public document to be released end of 2016. 